\title{
Exp-Function Method with Computerized Symbolic Computation for New Exact Solution of Burger's Equation
}

\author{
Sheng Zhang ${ }^{1, a}$, Qun Gao ${ }^{1, b}$ \\ ${ }^{1}$ School of Mathematics and Physics, Bohai University, Jinzhou, 121013, China \\ aemail: szhangchina@126.com, 'email: gaofeiqunjv@163.com
}

Keywords: Symbolic Computation; Exact Solution; Exp-Function Method; Burger's Equation

\begin{abstract}
It is well known that computer applications are ubiquitous. In this paper, the exp-function method with computerized symbolic computation is improved to exactly solve a (2+1)-dimensional Burger's equation. As a result, a new and more general exact solution is obtained. It is shown that the improved exp-function method can be used for other nonlinear partial differential equations (PDEs).
\end{abstract}

\section{Introduction}

Exactly solving nonlinear PDEs plays an important role in the study of nonlinear physical phenomena in many fields of nonlinear science. In the past several decades, some effective methods equipped with the powerful computerized symbolic computation systems like Mathematica or Maple have been proposed for nonlinear PDEs [1-8]. It is shown that these symbolic computation systems become indispensable auxiliary tools for solving complex PDEs or constructing more general solutions of PDEs. Among the existing methods, the exp-function method [1] proposed by $\mathrm{He}$ and $\mathrm{Wu}$ in 2006 is a simple and direct method for nonlinear PDEs. More importantly, the exp-function method and its improvements are available for many PDEs such as those in [9-16], this is due to the method possesses a more general ansätz [17]:

$$
u=\frac{\sum_{n=-f}^{g} a_{n} \exp (n \xi)}{\sum_{m=-p}^{q} b_{m} \exp (m \xi)}, \quad \xi=\sum_{i=1}^{s} k_{i} x+w t
$$

Supposed for a given nonlinear PDE with independent variables $t, x_{1}, x_{2}, \cdots, x_{s}$ and dependent variable $u$ :

$F\left(u, u_{t}, u_{x_{1}}, u_{x_{2}}, \cdots, u_{x_{m}}, u_{x_{1}}, u_{x_{2} t} \cdots, u_{x_{s}}, u_{t t}, u_{x_{1} x_{1}}, u_{x_{2} x_{2}}, \cdots, u_{x_{s} x_{s}}, \cdots\right)=0$.

In Eq. (1), $a_{n}$ and $b_{m}$ are undetermined constants, integers $f, p, g$ and $q$ can be determined by balancing the highest order linear term with the highest order nonlinear term in Eq. (2). In general, the final solution does not strongly depend on the choices of values of $f, p, g$, $q$ and $f=p=g=q=1$ is usually the simplest choice. In [17], Zhang et al. proposed a direct algorithm of the exp-function method for simplifying the computation so that the so-called middle expression expansion problem can be effectively solved. In this paper, we would like to improve the exp-function method by introducing the following general ansätz:

$$
u=\frac{\sum_{n=-f}^{g} a_{n}(y, z, \cdots, t) \exp (n \xi)}{\sum_{m=-p}^{q} b_{m}(y, z, \cdots, t) \exp (m \xi)}, \quad \xi=k x+\eta(y, z, \cdots, t),
$$

where $a_{n}(y, z, \cdots, t), \quad b_{m}(y, z, \cdots, t)$ and $\eta(y, z, \cdots, t)$ are undetermined differentiable functions of the indicated variables. To test the effectiveness and advantage of ansätz (3), in the next section we consider the following (2+1)-dimensional Burger's equation [18]:

$$
\left(u_{t}+u u_{x}-u_{x x}\right)_{x}+u_{y y}=0 .
$$




\section{Exact Solutions}

We suppose that

$$
u=\frac{a_{1}(y,, t) \exp (n \xi)+a_{0}(y, t)+a_{-1}(y, t) \exp (-n \xi)}{b_{1}(y, t) \exp (n \xi)+b_{0}(y, t)+b_{-1}(y, t) \exp (-n \xi)}
$$

where $\xi=k x+\eta(y, t), \quad a_{n}(y, t), \quad b_{m}(y, t)$ and $\eta(y, t)$ are all differentiable functions to be determined later. Substituting Eq. (5) into Eq. (4) and then equating each coefficient of the same order power of $\exp (j \xi)(j=1,2, \cdots, 8)$ to zero yields a system of nonlinear PDEs for $a_{1}(y, t)$, $a_{0}(y, t), \quad a_{-1}(y, t), \quad b_{1}(y, t), \quad b_{0}(y, t), \quad b_{-1}(y, t)$ and $\eta(y, t)$. From this derived system of nonlinear PDEs, we can see that if the solution (5) is a non-trivial one then the following equations must hold simultaneously

$$
a_{1 y y}(y, t)=0, \quad a_{-1 y y}(y, t)=0 .
$$

When $a_{0}(y, t)=0, \quad b_{1}(y, t)=b_{1}, \quad b_{0}(y, t)=0 \quad$ and $\quad b_{-1}(y, t)=b_{-1}$, here $b_{1}$ and $b_{-1}$ are arbitrary constants, the system of nonlinear PDEs are reduced as follows:

$$
\begin{aligned}
-8 b_{1}^{2} b_{-1} k^{3} a_{1}(y, t)-4 b_{1} b_{-1} k^{2} a_{1}^{2}(y, t)+8 b_{1}^{3} k^{3} a_{-1}(y, t)+4 b_{1}^{2} k^{2} a_{1}(y, t) a_{-1}(y, t) \\
+2 b_{1}^{2} b_{-1} k a_{1 t}(y, t)-2 b_{1}^{3} k a_{1 t}(y, t)-4 b_{1}^{2} b_{-1} k a_{1}(y, t) \eta_{t}(y, t)+4 b_{1}^{3} k a_{-1}(y, t) \eta_{t}(y, t) \\
+4 b_{1}^{2} b_{-1} a_{1 y}(y, t) \eta_{y}(y, t)-4 b_{1}^{3} a_{-1 y}(y, t) \eta_{y}(y, t)-4 b_{1}^{2} b_{-1} a_{1}(y, t) \eta_{y}^{2}(y, t) \\
+4 b_{1}^{3} a_{-1}(y, t) \eta_{y}^{2}(y, t)+2 b_{1}^{2} b_{-1} a_{1}(y, t) \eta_{y y}(y, t)-2 b_{1}^{3} a_{-1}(y, t) \eta_{y y}(y, t)=0, \\
32 b_{1} b_{-1}^{2} k^{3} a_{1}(y, t)+8 b_{-1}^{2} k^{2} a_{1}^{2}(y, t)-32 b_{1}^{2} b_{-1} k^{3} a_{-1}(y, t)-16 b_{1} b_{-1} k^{2} a_{1}(y, t) a_{-1}(y, t) \\
+8 b_{1}^{2} k a_{-1}^{2}(y, t)+4 b_{1} b_{-1}^{2} k a_{1 t}(y, t)-4 b_{1}^{2} b_{-1} k a_{-1 t}(y, t)+4 b_{1}^{2} b_{-1} k a_{-1 t}(y, t) \\
+8 b_{1}^{2} b_{-1} a_{1 y}(y, t) \eta_{y}(y, t)+4 b_{1} b_{-1}^{2} a_{1}(y, t) \eta_{y y}(y, t)-4 b_{1}^{2} b_{-1} a_{-1}(y, t) \eta_{y y}(y, t)=0, \\
-8 b_{-1}^{3} k^{3} a_{1}(y, t)+8 b_{1} b_{-1}^{2} k^{3} a_{-1}(y, t)+4 b_{-1}^{2} k a_{1 t}(y, t)-4 b_{1} b_{-1} k^{2} a_{-1}^{2}(y, t) \\
+2 b_{1}^{3} k a_{1 t}(y, t)-2 b_{1} b_{-1}^{2} k a_{1 t}(y, t)+4 b_{-1}^{3} k a_{1}(y, t) \eta_{t}(y, t)-4 b_{1} b_{-1}^{2} k a_{-1}(y, t) \eta_{t}(y, t) \\
+4 b_{-1}^{3} a_{1 y}(y, t) \eta_{y}(y, t)-4 b_{1} b_{-1}^{2} a_{-1 y}(y, t) \eta_{y}(y, t)+4 b_{-1}^{3} a_{1}(y, t) \eta_{y}^{2}(y, t) \\
-4 b_{1} b_{-1}^{3} a_{-1}(y, t) \eta_{y}^{2}(y, t)+2 b_{-1}^{3} a_{1}(y, t) \eta_{y y}(y, t)-2 b_{1} b_{-1}^{2} a_{-1}(y, t) \eta_{y y}(y, t)=0 .
\end{aligned}
$$

Solving Eqs. (6)-(9), we have

$$
a_{1}(y, t)=-b_{1}\left(1+2 k+\frac{f^{\prime}(t)}{k}\right), \quad a_{-1}(y, t)=-b_{-1}\left(1-2 k+\frac{f^{\prime}(t)}{k}\right), \quad \eta(y, t)=l y+f(t),
$$

where $l$ is an arbitrary constant, $k$ is a non-zero constant, and $f(t)$ is an arbitrary differentiable function of $t$.

With the help of Eqs. (5) and (10), we obtain a new exact and explicit solitary wave solution of Eq. (4):

$$
u=\frac{\left[-b_{1}\left(1+2 k+\frac{f^{\prime}(t)}{k}\right)\right] \exp (\xi)+\left[-b_{-1}\left(1-2 k+\frac{f^{\prime}(t)}{k}\right)\right] \exp (-\xi)}{b_{1} \exp (\xi)+b_{-1} \exp (-\xi)},
$$

where $\xi=k x+l y+f(t)$. It is easy to see that solution (11) cannot be constructed by the ansätz (1) used in the exp-function method [1]. Since the arbitrary function $f(t)$ is contained, solution (11) is more general than the one constructed by the exp-function method [1]. Besides, if we set $f(t)=w t$, here $w$ is a constant, then solution (11) becomes

$$
u=\frac{\left[-b_{1}\left(1+2 k+\frac{w}{k}\right)\right] \exp (\xi)+\left[-b_{-1}\left(1-2 k+\frac{w}{k}\right)\right] \exp (-\xi)}{b_{1} \exp (\xi)+b_{-1} \exp (-\xi)},
$$

where $\xi=k x+l y+w t$. Because solution (12) is a special case of solution (11), the improved exp-function method equipped with ansätz (3) has advantage over the exp-function method [1]. 
With the arbitrary function $f(t)$, solution (11) possesses rich spatial structures. For example, we set $f(t)=1-\operatorname{sn}\left(1+t^{2}, m\right), \quad k=1, \quad b_{1}=1$, and $b_{-1}=1$, a spatial structure of solution (11) is shown in Fig. 1. For the comparison between solutions (11) and (12), we use Fig. 2 to show a spatial structure of solution (12) by selecting $w=1$ and letting the other parameters be same as those in Fig. 1.

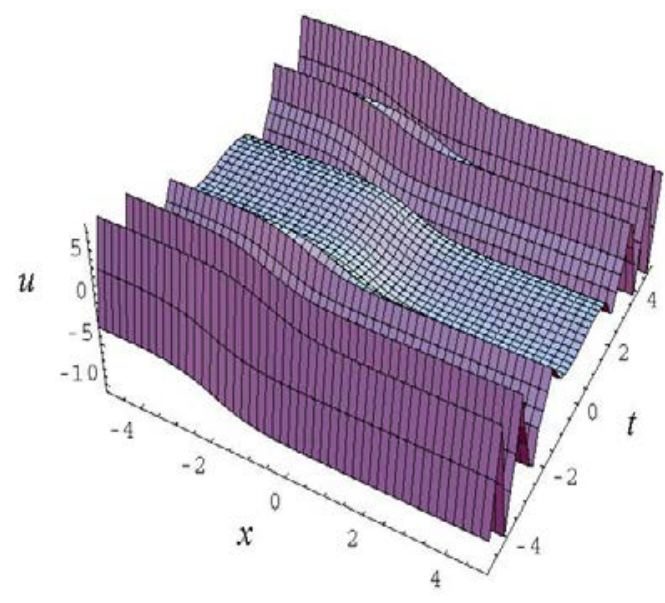

(a) $x \in[-5,5], \quad y=0, \quad t \in[-5,5]$

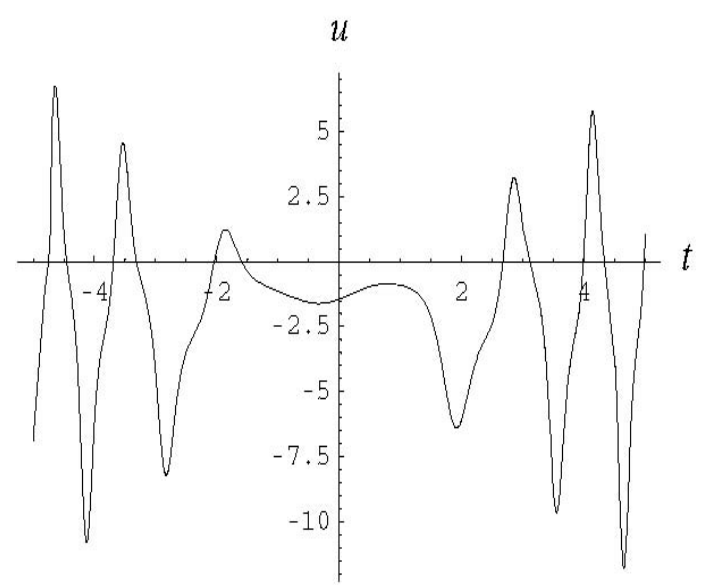

(b) $x=0, \quad y=0, \quad t \in[-5,5]$

Fig. 1. Spatial structure solution (11)

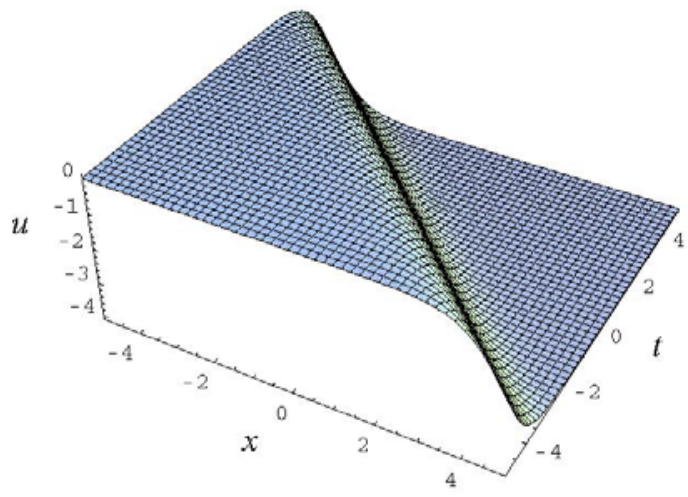

(a) $x \in[-5,5], \quad y=0, \quad t \in[-5,5]$

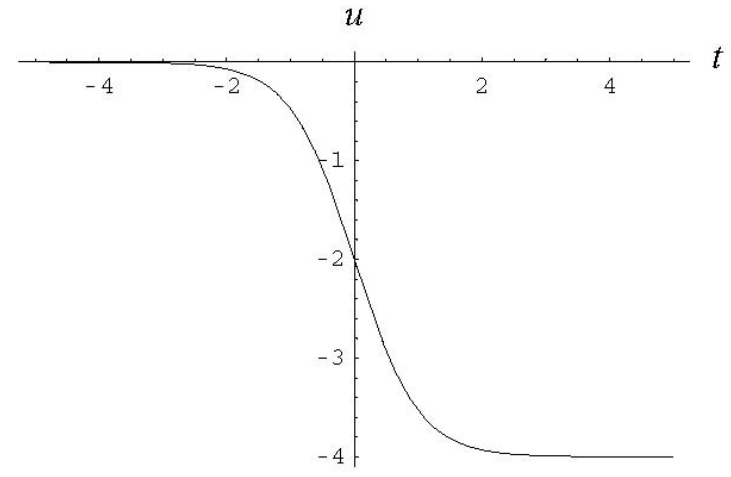

(b) $x=0, \quad y=0, \quad t \in[-5,5]$

Fig. 2. Spatial structure of solution (12)

From Figs. 1 and 2, we can see that solutions obtained by the improved exp-function method in present paper may have new spatial structures besides the spatial structures determined by the solutions constructed by the exp-function method [1].

\section{Conclusion}

In summary, we have improved the exp-function method by introducing ansätz (3) which includes the one used in the exp-function method [1] as a special case. Due to the generality of ansätz (3), new and more general solutions with arbitrary functions can be obtained. The effectiveness and advantage of the improved exp-function method have been tested through the $(2+1)$-dimensional Burger's equation. Applying the improved exp-function method presented in this paper to other nonlinear PDEs is worthy of study.

\section{Acknowledgement}

This work was supported by the Natural Science Foundation of China (11547005), the PhD Start-up Fund of Liaoning Province of China (20141137), the Liaoning BaiQianWan Talents Program (2013921055) and the Natural Science Foundation of Liaoning Province of China (L2012404). 


\section{References}

[1] Jihuan He, Xuhong Wu. Exp-function method for nonlinear wave equations. Chaos, Solitons and Fractals, 30 (2006) 700-708.

[2] Emmanuel Yomba. The modified extended Fan sub-equation method and its application to the (2+1)-dimensional Broer-Kaup-Kupershmidt equation. Chaos, Solitons and Fractals 27 (2007) 187-196.

[3] Sheng Zhang, Meitong Chen, Weiyi Qian. Painleve analysis for a forced Korteveg-de Vries equation arisen in fluid dynamics of internal solitary waves, Thermal Science, 19 (2015) 1223-1226.

[4] Mingliang Wang, Xiangzheng Li. Extended F-expansion method and periodic wave solutions for the generalized Zakharov equations. Physics Letters A, 343 (2005) 48-54.

[5] Sheng Zhang, Tiecheng Xia. A generalized auxiliary equation method and its application to (2+1)-dimensional asymmetric Nizhnik-Novikov-Vesselov equations, Journal of Physics A: Mathematical and Theoretical, 40 (2006) 227-248

[6] Sheng Zhang, Xudong Gao. Exact N-soliton solutions and dynamics of a new AKNS equations with time-dependent coefficients, Nonlinear Dynamics, 83(2016) 1043-1052.

[7] Engui Fan. Travelling wave solutions in terms of special functions for nonlinear coupled evolution systems. Physics Letters A, 300 (2002) 243-249.

[8] Sheng Zhang, Hongqing Zhang. A transformed rational function method for (3+1)-dimensional potential YTSF equation. Pramana-Journal of Physics, 76 (2011) 561-571.

[9] Sheng Zhang, Application of exp-function method to a $\mathrm{KdV}$ equation with variable coefficients. Phys. Lett. A 365 (2007) 448-453

[10] Limei Yan. Generalized exp-function method for non-linear space-time fractional differential equations. Thermal Science, 18 (2014) 1573-1576.

[11] Sheng Zhang. Exact solutions of a KdV equation with variable coefficients via exp-function method. Nonlinear Dynamics, 52 (2008) 11-17.

[12] Sheng Zhang. Application of exp-function method to high-dimensional nonlinear evolution equation. Chaos, Solitons and Fractals, 38 (2008) 270-276.

[13] Jihuan He, Lina Zhang. Generalized solitary solution and compacton-like solution of the Jaulent-Miodek equations using the exp-function method. Physics Letters A, 372 (2008) 1044-1047.

[14] Sheng Zhang, Hongqing Zhang. Exp-function method for $N$-soliton solutions of nonlinear evolution equations in mathematical physics. Physics Letters A, 373 (2009) 2501-2505.

[15] Sheng Zhang. Exp-function method for Riccati equation and new exact solutions with two arbitrary functions of (2+1)-dimensional Konopelchenko-Dubrovsky equations. Applied Mathematics and Computation, 216 (2010) 1546-1552.

[16] Sheng Zhang, Jian Wang, Aoxue Peng, Bin Cai. A generalized exp-function method for multiwave solutions of sine-Gordon equation. Pramana-Journal of Physics, 81 (2013) 763-77.

[17] Sheng Zhang, Jiahong Li, Luyao Zhang. A direct algorithm of exp-function method for nonlinear evolution equations in fluids. Thermal Science, 20 (2016) 881-884.

[18] Boling Guo, Xiaofeng Pang. Solitons, Beijging: Science Press, 1987. 